\title{
Frequency-doubled scattering of symmetry-breaking surface-state electrons on liquid Helium
}

\author{
Miao Zhang, ${ }^{1}$ Wenzhi Jia, ${ }^{1}$ and Lianfu Wei ${ }^{1,2 *}$ \\ ${ }^{1}$ Department of Physics, Southwest Jiaotong University, Chengdu 610031, China \\ ${ }^{2}$ State Key Laboratory of Optoelectronic Materials and Technologies, \\ School of Physics and Engineering, Sun Yat-sen University, Guangzhou 510275, China \\ *Corresponding author: weilianfu@gmail.com
}

\begin{abstract}
Compiled January 6, 2018
Any systems with symmetry-breaking eigenstates can effectively radiate photons with doubled frequency of the incident light, which is known as the second harmonic generation. Here, we study the second-order nonlinear effects with the system of surface-state electrons on liquid Helium. Due to the symmetry-breaking eigenstates, we show that a Rabi oscillation between two levels of the surface-state electrons can be realized beyond the usual resonant driving. Consequently, an electromagnetic field with the doubled frequency of the applied driving could be effectively radiated. This can be regarded as a frequency-doubled fluorescence, and interestingly, it works in the unusual Terahertz range. (C) 2018 Optical Society of America

OCIS codes: 270.4180, 300.2530.
\end{abstract}

Introduction. - An electron (with mass $m_{e}$ and charge e) near the surface of liquid Helium is weakly attracted by its dielectric image potential $V(z)=-\Lambda e^{2} / z$. Where $z$ is the distance above liquid Helium surface, and $\Lambda=$ $(\varepsilon-1) / 4(\varepsilon+1)$ with $\varepsilon$ being dielectric constant of the liquid Helium [1]. Due to the Pauli exclusion principle, there is an barrier about $1 \mathrm{eV}$ for preventing the electron penetrating into liquid Helium. Together with such a hard wall at $z=0$, the electron is resulted in an onedimensional (1D) hydrogenlike spectrum $E_{n}=-R / n^{2}$ of motion normal to the Helium surface, with Rydberg energy $R=\Lambda^{2} e^{4} m_{e} /\left(2 \hbar^{2}\right) \approx 8 \mathrm{~K}$ and effective Bohr radius $r_{b}=\hbar^{2} /\left(m_{e} e^{2} \Lambda\right) \approx 76 \AA$. These Rydberg levels were first observed by Grimes et al. $[1,2]$ by measuring the resonant frequencies of the transitions between the ground and excited states. Recently, it has been studied that the above Rydberg states have relatively-long lifetimes [3], and possibly, the electrons could be used as qubits (with the ground and first excited states) to realize the relevant quantum information processing [4-10].

In this letter, we first study the second-order nonlinear optical effect of the surface-state electrons on liquid Helium. Due to the symmetry-breaking surface-states, the electrons allow an unusual dipole moment which is related to the nonzero average distances between the Rydberg states, i.e., $d=\langle m|z| m\rangle-\langle n|z| n\rangle \neq 0$. Basically, under the resonant driving the usual transition $\langle n|z| m\rangle$ is dominant and the effects related to $d$ are negligible. Alternatively, under the certain large-detuning driving, the effects related to $d$ become important, and consequently a Rabi oscillation between two selected levels of the surface-state electrons could be realized beyond the usual resonant driving. This Rabi oscillation leads an electromagnetic wave emission with the doubled frequency of the applied driving.

Rabi oscillations beyond the resonant driving.-We consider applying a microwave field $E_{l}=\mathcal{E} \cos \left(\omega_{l} t-k r+\right.$ $\phi)$ to a single surface-state electron, where $\mathcal{E}, \omega_{l}, k$, and $\phi$ are its amplitude (in $z$ direction), frequency, wave vector, and initial phase, respectively. For simplicity, we consider only two levels (i.e., the ground state $|1\rangle$ and the first excited state $|2\rangle)$ of the surface-state electron. Therefore, under the usual dipole approximation $(k r \approx 0)$ the Hamiltonian describing the driven two-level electron reads $\hat{H}(t)=E_{1}|1\rangle\left\langle 1\left|+E_{2}\right| 2\right\rangle\langle 2|-e \hat{z} \mathcal{E} \cos \left(\omega_{l} t+\phi\right)$. By using the completeness relation $|1\rangle\langle 1|+| 2\rangle\langle 2|=\hat{\mathbf{1}}$, one can write $\hat{z}$ as $\hat{z}=z_{11}|1\rangle\left\langle 1\left|+z_{22}\right| 2\right\rangle\left\langle 2\left|+z_{12}\right| 1\right\rangle\langle 2|+$ $z_{21}|2\rangle\langle 1|$ with the dipole matrix elements $z_{12}=\langle 1|\hat{z}| 2\rangle=$ $\langle 2|\hat{z}| 1\rangle=z_{21}$. Due to the asymmetry eigenstates $|1\rangle$ and $|2\rangle$, here $z_{11}=\langle 1|\hat{z}| 1\rangle$ and $z_{22}=\langle 2|\hat{z}| 2\rangle \neq 0$. This is very different from the case for the natural atoms wherein $z_{11}=z_{22}=0$.

Obviously, the Hamiltonian of the electron can be rewritten as

$\hat{H}(t)=\frac{\hbar \omega_{e}}{2} \hat{\sigma}_{z}-\frac{1}{2}\left[\hbar \widetilde{\Omega} \hat{\sigma}_{z}+\hbar \Omega_{\mathrm{R}}\left(\hat{\sigma}_{12}+\hat{\sigma}_{21}\right)\right] \cos \left(\omega_{l} t+\phi\right)$,

and further

$$
\begin{aligned}
\hat{H}(t)= & -\hbar \widetilde{\Omega} \hat{\sigma}_{z}\left(e^{i \omega_{l} t+i \phi}+\text { H.c }\right) \\
& -\hbar \Omega_{\mathrm{R}}\left[\hat{\sigma}_{12}\left(e^{-i \Delta t+i \phi}+e^{-i\left(\omega_{e}+\omega_{l}\right) t-i \phi}\right)+\text { H.c. }\right],
\end{aligned}
$$

in the interaction picture defined by $\hat{R}=$ $\exp \left(-i t \omega_{e} \hat{\sigma}_{z} / 2\right)$. Above, $\hat{\sigma}_{z}=|2\rangle\langle 2|-| 1\rangle\langle 1|$ is the Pauli operator with the transition frequency $\omega_{e}=\left(E_{2}-E_{1}\right) / \hbar$, $\hat{\sigma}_{i j}=|i\rangle\langle j|$ is the projection operator (with $i, j=1,2$ ), $\Delta=\omega_{e}-\omega_{l}$ is the detuning between the microwave and electron, $\Omega_{\mathrm{R}}=z_{12} e \mathcal{E} /(2 \hbar)$ is the usual Rabi frequency, and finally $\widetilde{\Omega}=\left(z_{22}-z_{11}\right) e \mathcal{E} /(4 \hbar) \neq 0$ due to the symmetry-breaking in the states $|1\rangle$ and $|2\rangle$.

Near the resonant point, i.e., $\Delta \ll\left(\omega_{l}, \omega_{e}\right)$, the evolution ruled by the Hamiltonian (2) can be approximately expressed as $\hat{U}(t)=1+\left(\frac{-i}{\hbar}\right) \int_{0}^{t} \hat{H}\left(t_{1}\right) d t_{1}+\cdots \approx$ 
$\hat{T} \exp \left[\int_{0}^{t} \hat{H}_{\mathrm{R}}(t) d t\right]$ with the Dyson-series operator $\hat{T}$ and effective Hamiltonian $\hat{H}_{\mathrm{R}}(t)=-\hbar \Omega_{\mathrm{R}}\left(e^{-i \Delta t+i \phi} \hat{\sigma}_{12}+\right.$ H.c.). In the rotating-framework defined by the unitary operator $\hat{R}_{\mathrm{R}}=\exp \left(i t \Delta \hat{\sigma}_{z} / 2\right)$, this effective Hamiltonian reads

$$
\hat{H}_{\mathrm{R}}=\frac{\hbar \Delta}{2} \hat{\sigma_{z}}-\hbar \Omega_{\mathrm{R}}\left(e^{i \phi} \hat{\sigma}_{12}+e^{-i \phi} \hat{\sigma}_{21}\right) .
$$

Certainly, when $\Delta=0$, the Hamiltonian (3) describes the standard Rabi oscillation with the frequency $\Omega_{\mathrm{R}}$. Above, the terms related to $\xi=\left(\Omega_{\mathrm{R}}, \widetilde{\Omega}\right) /\left(\omega_{l}, \omega_{e}\right)$ are neglected, since $\xi \ll 1$ under the weak drivings. In fact, this approximation is nothing but the usual rotating-wave approximation. Indeed, under the resonant excitation, i.e., $\Delta \ll\left(\omega_{l}, \omega_{e}\right)$, the contribution from $\hbar \widetilde{\Omega} \hat{\sigma}_{z}$ (due to the symmetry-breaking of the present surface-state electrons) should be negligible, due to its small probability proportional to $\xi$.

Interestingly, under the large-detuning driving, e.g., $\Delta=\omega_{l}-\delta$ (with a small adjustment $\delta \sim 0$ ), the effects related to the Stark term $\hbar \widetilde{\Omega} \hat{\sigma}_{z}$ become significant. This can be seen from the following new evolution operator [11]

$$
\begin{aligned}
\hat{U}(t)= & 1+\left(\frac{-i}{\hbar}\right) \int_{0}^{t} \hat{H}\left(t_{1}\right) d t_{1} \\
& +\left(\frac{-i}{\hbar}\right)^{2} \int_{0}^{t} \hat{H}\left(t_{1}\right) \int_{0}^{t_{1}} \hat{H}\left(t_{2}\right) d t_{2} d t_{1}+\cdots \\
\approx & \hat{T} \exp \left[\int_{0}^{t} \hat{H}_{\mathrm{L}}(t) d t\right]
\end{aligned}
$$

with the second-order effective Hamiltonian $\hat{H}_{\mathrm{L}}(t)=$ $\hbar \nu \hat{\sigma}_{z} / 2-\hbar \Omega_{\mathrm{L}}\left(e^{i \delta t+i 2 \phi} \hat{\sigma}_{12}+\right.$ H.c. $)$, which can be further written as

$$
\hat{H}_{\mathrm{L}}=\frac{\hbar \Delta^{\prime}}{2} \hat{\sigma}_{z}-\hbar \Omega_{\mathrm{L}}\left(e^{i 2 \phi} \hat{\sigma}_{12}+e^{-i 2 \phi} \hat{\sigma}_{21}\right)
$$

in the rotating framework defined by $\hat{R}_{\mathrm{L}}=$ $\exp \left(-i t \delta \hat{\sigma}_{z} / 2\right)$. Obviously, if the "new detuning" $\Delta^{\prime}=0$, i.e., $\omega_{l} \approx \omega_{e} / 2$, the Hamiltonian (5) also defines to a Rabi oscillation with the frequency $\Omega_{\mathrm{L}}$.

Above, the value of $\delta=2 \omega_{l}-\omega_{e}$ is controllable by the selected frequency $\omega_{l}$ of the applied microwave, and $\Delta^{\prime}=\nu-\delta$ with $\nu=4 \Omega_{\mathrm{R}}^{2}\left[1 /\left(\omega_{e}-\delta\right)+1 /\left(3 \omega_{e}+\delta\right)\right]$. Due to the weak driving $\left(\Omega_{\mathrm{R}}, \widetilde{\Omega}\right) \ll\left(\omega_{l}, \omega_{e}\right)$, the present Rabi frequency $\Omega_{\mathrm{L}}=4 \Omega_{\mathrm{R}} \widetilde{\Omega} \omega_{e} /\left(\omega_{e}^{2}-\delta^{2}\right)$ is smaller than the previous one $\Omega_{\mathrm{R}}$ for the resonant excitation. Also, all the effects related to the small quantity $\xi=\left(\Omega_{\mathrm{R}}, \widetilde{\Omega}\right) /\left(\omega_{l}, \omega_{e}\right)$ were neglected, but the terms containing $\Omega_{\mathrm{R}} \widetilde{\Omega} /\left(\omega_{l}, \omega_{e}\right)$ were retained. Note that, under the above large-detuning driving, the usual first-order expansion term $\hat{U}^{(1)}(t)=$ $(-i / \hbar) \int_{0}^{t} \hat{H}\left(t_{1}\right) d t_{1}$ practically does not contribute to the time evolution, due to its small probability: $P^{(1)}(t) \sim \xi$.

Frequency-doubling radiations. - We now consider the steady-state radiations due to the above oscillations of occupancies by taking into account the practicallyexisting dissipations. For this case the dynamics of the

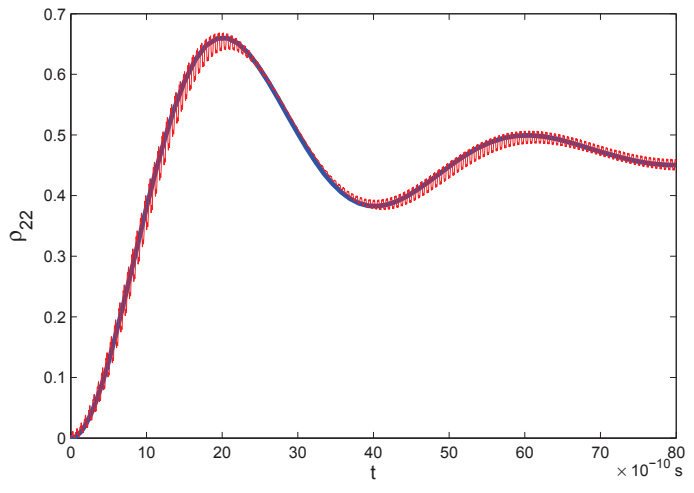

Fig. 1. (Color online) Time dependent $\rho_{22}$, with $\Delta^{\prime}=0$ and $\Gamma=\gamma=g / 10$. The blue and red lines correspond to the analytical solution from the effective Hamiltonian (5) and the numerical one from the original Hamiltonian (1), respectively. The relevant parameters are selected as $[2,5]: \omega_{e} \approx 0.22 \mathrm{THz}, z_{12} \approx 0.5 r_{b}, z_{22}-z_{11} \approx 2.3 r_{b}$, and $\mathcal{E} \approx 15 \mathrm{~V} / \mathrm{cm}$, and consequently $\Omega_{\mathrm{R}} \approx 4.3 \mathrm{GHz}$ and $\Omega_{\mathrm{L}} \approx 0.8 \mathrm{GHz}$.

driven surface-state electrons is described by the master equation

$$
\begin{aligned}
\frac{d \hat{\rho}}{d t}= & \frac{-i}{\hbar}\left[\hat{H}_{\mathrm{L}}, \hat{\rho}\right]+\frac{\Gamma}{2}\left(2 \hat{\sigma}_{12} \hat{\rho} \hat{\sigma}_{21}-\hat{\sigma}_{21} \hat{\sigma}_{12} \hat{\rho}-\hat{\rho} \hat{\sigma}_{21} \hat{\sigma}_{12}\right) \\
& +\frac{\gamma}{2}\left(2 \hat{\sigma}_{22} \hat{\rho} \hat{\sigma}_{22}-\hat{\sigma}_{22} \hat{\rho}-\hat{\rho} \hat{\sigma}_{22}\right)
\end{aligned}
$$

with $\Gamma$ and $\gamma$ being the decay and dephasing rates, respectively. $\hat{\rho}=\sum_{i, j=1}^{2} \rho_{i j}|i\rangle\langle j|$ is the density operator of the two-level quantum system, and the matrix elements $\left\{\rho_{i j}\right\}$ obey the normalized and hermitian conditions: $\sum_{i=1}^{2} \rho_{i i}=1$ and $\rho_{i j}=\rho_{j i}^{*}$, respectively. Certainly, due to the resistance from the surroundings, the amplitudes of oscillating $\rho_{i i}$ decrease with the time evolving (see, e.g., Fig. 1), and $\dot{\rho}_{i i} \rightarrow 0$ when $t \rightarrow \infty$. Specially, under the steady-state condition: $\dot{\rho}_{i j}=0$, we have $\rho_{22}=2 \Omega_{\mathrm{L}}^{2} \kappa /\left[\Gamma\left(\kappa^{2}+\Delta^{\prime 2}\right)+4 \Omega_{\mathrm{L}}^{2} \kappa\right]$ and $\rho_{21}=$ $\Omega_{\mathrm{L}} e^{-i 2 \phi}\left(\rho_{11}-\rho_{22}\right)\left(i \kappa+\Delta^{\prime}\right) /\left(\kappa^{2}+\Delta^{\prime 2}\right)$ with $\kappa=(\Gamma+\gamma) / 2$.

Immediately, we can compute the polarization $P=$ $\langle e z\rangle=\operatorname{Tr}(e \hat{\rho} \hat{z})=e \sum_{i, j=1}^{2} \rho_{i j} z_{j i}$ for single surfacestate electrons. Note that the Eq. (5) is obtained in the rotating-framework with frequency $\omega_{e}+\delta=2 \omega_{l}$. Transforming back to the Schrödinger picture, the polarization of the electrons reads

$$
P=e\left(\rho_{21} z_{12} e^{-i 2 \omega_{l} t}+\text { H.c. }\right)+e\left(\rho_{11} z_{11}+\rho_{22} z_{22}\right) .
$$

This indicates that the surface-state electron has a steady $2 \omega_{l}$-response for the applied incident field of frequency $\omega_{l}$. Such a $2 \omega_{l}$-response acts further as an effective source of new radiation with doubled-frequency $2 \omega_{l}$. By discarding the static polarizations $\rho_{11} z_{11}$ and $\rho_{22} z_{22}$ (which practically does not contribute to the generations of the radiations), we can write the polarization (7) in a simple form $P=A \cos \left(2 \omega_{l} t-\theta\right)$ with the amplitude $A=e z_{12} \sqrt{\rho_{21} \rho_{12}}$ and phase $\theta=\arctan \left(\operatorname{Im} \rho_{21} / \operatorname{Re} \rho_{21}\right)+$ $2 \phi$. 


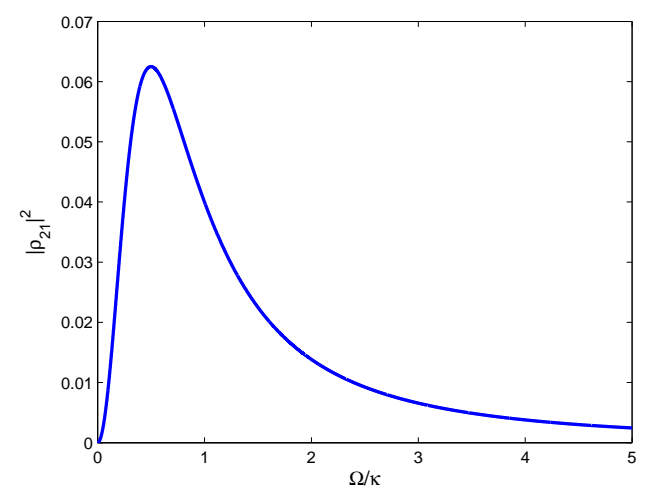

Fig. 2. (Color online) Scattering saturation: $\left|\rho_{21}\right|^{2}$ versus $\Omega / \kappa$ with typical $\kappa=\Gamma$ and $\mathcal{D}=0$. If $\Omega=\Omega_{\mathrm{R}}$ and $\mathcal{D}=\Delta$ this figure corresponds to that of the usual resonant scattering, and if $\Omega=\Omega_{\mathrm{L}}$ and $\mathcal{D}=\Delta^{\prime}$ the figure corresponds to that of the present frequency-doubled scattering.

Obviously, the radiating power of this electric-dipole reads $I=A^{2}\left(2 \omega_{l}\right)^{4} /\left(12 \pi \epsilon_{0} c^{3}\right)$, which is proportional to $\left|\rho_{21}\right|^{2}$. In the weak field regime, i.e., $\Omega_{\mathrm{L}} \ll \kappa$, the microwave scattering can be significantly enhanced by increasing $\Omega_{\mathrm{L}}$ as shown in Fig. 2. However, if $\Omega_{\mathrm{L}}$ is further enlarged, the scattering appears saturation and begins to decrease. Note that the Hamiltonian (3) (for resonant exciting) and the Hamiltonian (5) (for large-detuning exciting) have the same form, and thus the present frequencydoubled scattering has the same peak value to that of the usual resonant scattering.

Discussions and Conclusions. - As the frequency of microwaves is relatively-low (compared to the usual optical frequency), the resonant or frequency-doubled scattering effect of the single surface-state electron is very weak, e.g., the induced $I \approx 10^{-25}$ W for the typical $\omega_{l}=0.22 \mathrm{THz}[2], z_{12} \approx 0.5 r_{b}$, and $\left|\rho_{21}\right|^{2}=0.063$. To significantly enhance the scattering power an effective approach is to enlarge the transition frequency $\omega_{e}$ (since $I \propto \omega_{e}^{4}$ ). For example, if the transition frequency is controlled as $\omega_{e}=2.7 \mathrm{THz}$, then the emission power of a single electron can reach $I \approx 2.2 \times 10^{-21} \mathrm{~W}$ (i.e., about one photons per second). This enhanced frequency can be achieved by applying a vertical electrostatic field $E_{\perp} \approx 0.91 \mathrm{kV} / \mathrm{cm}$ to the electron [5]. Of course, the scattered power can also be enhanced by increasing the number of electrons. For the multi-electrons system, we assume that: (1) the microwave-absorption is sufficiently small such that the amplitude of the applied microwave can be regarded unchanged during microwave-electron interaction, (2) the electron-electron Coulomb interaction [12] and the sub-drivings of the scattering waves are negligible, i.e, they are far weaker than the driving of the applied microwave and the decay of electrons. Therefore, the total scattering power of $n$ electrons can be simply expressed as $I_{t} \approx n I$. In the experiment [3], the electrons are confined in an area $s \approx 5 \mathrm{~cm} \times 5 \mathrm{~cm}$ with a low electron-density of $\eta \approx 0.18 \times 10^{11} \mathrm{~m}^{-2}$. With these typical parameters, we have $I_{t} \approx s \eta I \approx 0.1 \mathrm{pW}$ for $\omega_{e}=2.7 \mathrm{THz}$, which could be observed by the $\mathrm{THz}$ detectors, see, e.g., [13, 14].

In conclusion, we have shown that an effective Rabi oscillation of the surface-state electrons on liquid Helium can be implemented under the large-detuning driving, i.e., $\omega_{l} \approx \omega_{e} / 2$. Based this Rabi oscillation, we have discussed the polarization (and consequently the frequencydoubled radiation) according to the steady-state solution of the relevant master equation. It has been shown that the present doubled-scattering has the same peak power to that of the usual resonant scattering. Experimentally, the scattered power of the single electron is very weak because of the lower microwave frequency. However, by enlarging the transition frequency and increasing the number of electrons the scattering power can be significantly enhanced and could be detected experimentally.

This work was supported by the National Natural Science Foundation of China Grants No. 11204249, 11147116, 11174373, and 90921010, the Major State Basic Research Development Program of China Grant No. 2010 CB923104.

\section{References}

1. C. C. Grimes and T. R. Brown, Phys. Rev. Lett. 32, 280 (1974).

2. C. C. Grimes, T. R. Brown, M. L. Burns, and C. L. Zipfel, Phys. Rev. B 13, 140 (1976).

3. E. Collin, W. Bailey, P. Fozooni, P. G. Frayne, P. Glasson, K. Harrabi, M. J. Lea, and G. Papageorgiou, Phys. Rev. Lett. 89, 245301 (2002).

4. P. M. Platzman and M. I. Dykman, Science 284, 1967 (1999).

5. M. I. Dykman, P. M. Platzman, and P. Seddighrad, Phys. Rev. B 67, 155402 (2003).

6. F. R. Bradbury, M. Takita, T. M. Gurrieri, K. J. Wilkel, K. Eng, M. S. Carroll, and S. A. Lyon, Phys. Rev. Lett. 107, 266803 (2011).

7. G. Papageorgiou, P. Glasson, K. Harrabi, V. Antonov, E. Collin, P. Fozooni, P. G. Frayne, M. J. Lea, and D. G. Rees, Appl. Phys. Lett. 86, 153106 (2005).

8. S. A. Lyon, Phys. Rev. A 74, 052338 (2006).

9. S. Mostame and R. Schützhold, Phys. Rev. Lett. 101, 220501 (2008).

10. D. I. Schuster, A. Fragner, M. I. Dykman, S. A. Lyon, and R. J. Schoelkopf, Phys. Rev. Lett. 105, 040503 (2010).

11. F. Deppe, M. Mariantoni, E. P. Menzel, A. Marx, S. Saito, K. Kakuyanagi, H. Tanaka, T. Meno, K. Semba, H. Takayanagi, E. Solano, and R. Gross, Nature Physics 4, 686 (2008).

12. D. Konstantinov, M. I. Dykman, M. J. Lea, Y. Monarkha, and K. Kono, Phys. Rev. Lett. 103, 096801 (2009).

13. S. Komiyama, O. Astafiev, V. Antonov, T. Kutsuwa, and H. Hirai, Nature 403, 405-407 (2000).

14. S. Komiyama, IEEE Journal of Selected Topics in Quantum Electronics 17, 54-66 (2011). 